\title{
In-network Processing for Context-Aware SOA-based Manufacturing Systems
}

\author{
Hasan Derhamy, Jens Eliasson, Jerker Delsing, Jan van Deventer \\ Dept of Computer Science, Electrical and Space Engineering \\ Luleå University of Technology, Luleå, Sweden. \\ Email:name.name@ltu.se
}

\begin{abstract}
To achieve flexible manufacturing, increasingly large amounts of data are being generated, stored, analyzed, archived and eventually fed back into the product life cycle. But where is this data stored and how is it transported? Current methods rely on centralized or federated databases to manage the data storage. This approach has several challenges, such as collection bottlenecks, secure retrieval, single point of failure and datascheme fragility as data heterogeneity increases. Additionally, manufacturers are finding the need to open their networks for service based equipment suppliers. This means previous security assumptions regarding network encryption and information access-control must be re-evaluated.

Proposed here is a method of in-network processing that gathers information only where and when it is needed. Systems build context at runtime by creating dynamic queries which make service composition. The service composition processes raw data and presents it as information to the calling system. This reduces the movement of data/information and removes single point collection bottlenecks. Furthermore, fine grained access control and shared trust can be granted between untrusted systems. The proposed methods are demonstrated on a lab setup of an industrial use case.
\end{abstract}

\section{INTRODUCTION}

Industry is demanding more Flexible, Efficient and Sustainable (FES) methods for manufacturing [1]. Factory digitization was seen as the next step forward. ISA 95 assisted factories in moving data between the factory floor and enterprise systems. However, due to pure vertical integration [2], information flow and communication in the horizontal and diagonal directions was not considered. In this pursuit, the Industry 4.0 initiative has generated the Reference Architectural Model for Industry 4.0 (RAMI 4.0) and the I4.0 component model [3]. They envisage communication between smart equipment and products that are wrapped in administrative shells. The FES gains require a change to many security assumptions and security objectives. This is to enable communication, co-operation and information flow between multi-stakeholder Systems of Systems. Information is a key enabler to finding efficiency gains and improving sustainable processes.

Raw data is gathered from IT systems and factory automation systems and stored in databases. Raw data has limited value and must be processed before its value can be realized. However processing the data into useful information can be costly and time consuming. Furthermore, even once improvements are identified, the software and automation systems are often brittle and resist this change.
Flexible manufacturing means that a production line is able to adapt rapidly to changes in the production process. This means that a customized order of lot size 1 is a unique product/variant. So a single production line must handle multiple products or variants at once. Managing the order/specification information and then disseminating it to the right automation system at the right time requires significant synchronization.

\section{A. Gap}

Current solutions to data extraction, analysis and dissemination either suffer from centralized bottlenecks and single points of failure or fixed/rigid middleware. An information exchange solution must conform the requirements:

Table I

THE REQUIREMENTS FOR INFORMATION EXCHANGE IN NEXT GENERATION INDUSTRIAL MANUFACTURING.

\begin{tabular}{|l|l|}
\hline Requirement & Description \\
\hline $\begin{array}{l}\text { Dynamic routing of } \\
\text { data between systems }\end{array}$ & $\begin{array}{l}\text { Systems a moving, changing and adapting, so } \\
\text { static routing of data is not flexible. }\end{array}$ \\
\hline $\begin{array}{l}\text { On-demand access to } \\
\text { information }\end{array}$ & $\begin{array}{l}\text { Mass collection/processing of data is not effi- } \\
\text { cient and secure. On-demand means information } \\
\text { made available only when and where it is } \\
\text { needed. }\end{array}$ \\
\hline $\begin{array}{l}\text { Controlled distribution } \\
\text { of data and } \\
\text { information }\end{array}$ & $\begin{array}{l}\text { Granular access rights and ownership of data/in- } \\
\text { formation must be supported. }\end{array}$ \\
\hline
\end{tabular}

Existing solutions using centralized data collection and processing or enforced middleware lack flexibility and are brittle to change.

The proposed solution is dynamic in nature, supports privacy-by-design and fine-grained access control through supporting systems. The proposed solution presented in Sections III and IV enables context-aware industrial systems by allowing information query from SOA-based networks without reliance on centralized data collection. This is implemented in a lab setup of an industrial use case, condition monitoring of a wheel loader ball bearing.

\section{B. Contribution}

Here we propose an information extraction architecture that provides in-network processing for context-aware systems. It builds on SOA-based principles. To achieve this there are three main challenges:

1) Language to express interest in certain information 
2) Orchestration method to parse the interest and identify the correct composition

3) Services available for processing raw data into presentable information

This paper tackles the first and third challenge. The second challenge is to be tackled in future work.

The first challenge requires a method of expressing interest for information. An interest must be able to express the required type of information (indicating the source of the data) and the functional transformations of data. We propose to use Cypher [4], an SQL inspired, graph query language. Section II-C details the expression of interest

Dynamic services are proposed to assist with standard data transformations. This includes thresholding trigger, data aggregation, Filter and etc. Dynamic services are service instances which are provisioned based on composition instructions. Section IV describes the details.

Orchestration for SOA-based systems builds on concepts introduced by Arrowhead Framework [5]. This paper assumes that composition facility is available.

The following sections will introduce the background to the importance of information exchange and its application and the related work.

\section{BACKGROUND}

\section{A. Context-awareness}

Context-aware computing starting in the 1990s focused primarily on providing location dependent information to mobile users. According to Abowd and Dey [6] context can be defined as:

"Context is any information that can be used to characterize the situation of an entity. An entity is a person, place, or object that is considered relevant to the interaction between a user and an application, including the user and applications themselves".

At its essence context-aware computing involves systems modifying their behavior based on changes to context over time. It is primarily used to decide what information to present to a user. For example a shopping list system may remind a user about the shopping list as they travel toward or past a supermarket. In this case, the system monitored the users context.

However context-aware computing when applied to Industrial Internet can enable advanced machine-to-machine behavior changes. Location is one aspect for a mobile system within a factory floor. However, a full list of relevant context might incorporate: 1) Production recipe, 2) Available machinery, 3) Available material 4) Operator synchronization - safety, validation, 5) Specifications, 6) Environment - temperature, humidity, 7) Location.

A context-aware industrial system interacts with other systems without user interaction. In this machine-to-machine environment, context moves in both vertical and horizontal manners. Overall production objectives and methods are based on planning and production recipes. While devices and product operation will generate context moving upward.
Context-aware systems decide the order of process execution based on available machinery. They do not begin dangerous processes while safe operation is not possible. A customer specification decides the quality and customizes a product recipe. Environmental factors affect quality, a context aware system do not begin a process before the environmental requirements are satisfied.

Many automatic control systems such as ABS braking react to input and could therefore be considered contextaware systems. These embedded systems fit the pervasive ubiquitous notion. They are single purpose tailored systems. However, these systems collect data, process it, and make decisions within a single system. Industrial context-aware systems cannot collect all raw data and process it themselves. This would result in much duplicate collection and processing of data. Rather they gather context based on responses from other systems in the service network.

\section{B. Related work}

Traditional approaches utilize centralized strategies with push based "command center" synchronization. In this case a number of enterprise databases connected by a software service bus are used to collect and disseminate factory information. If there are any issues with the service bus, an entire production lines can suffer reduced performance if not complete breakdown. This risk means that data access by nonproduction related activities are restricted and minimized. This further reduces access to the raw data for processing by quality or production engineering, looking to measure improvements or find issues.

Context-aware systems are systems that utilize knowledge about their environment and self driven objectives in their decision making. Flexible manufacturing processes use smart products/equipment that are able to question their environment and build a life cycle understanding. It can then make decisions about: 1) what is the next step in its process; 2) when it can execute the next step; 3) raise alerts regarding quality assurance; 4) make use of surrounding services; In this way, a flexible manufacturing line will have many context-aware systems that make varied decisions as the context changes. Complex operations emerge from simple actions. Once again, the issue of information management raises questions of scalability, security and changeability.

In-network processing as described by Tannenbaum [7] is an approach of treating the computer network as a means of processing raw data and aggregating or presenting the information to the requester. TinyDB [8] is an example of innetwork processing applied to wireless sensor networks. But it can also be applied to general computing. Any network can act as a database that can be queried for information that may not exist until the query is processed. One of the benefits of in-network processing are to avoid network and memory overheads of centralizing data and information. Furthermore, by aggregating and preparing results, it can help reduce the complexity systems consuming data from multiple sources. 
Another similar approach to in-network processing is Information centric networking (ICN). It is a proposal to move from host based addressing to information name addressing [9]. Regardless of where the information may exist, a system sends out an "interest" for a particular information. This "interest" is then spread throughout the information centric network until the information is found and returned to the requester. An extension to ICN is Service Centric Networking (SCN) [10]. SCN extends ICN to include in-network functions that process existing data/information into new information. This is a recursive operation where information $=f$ (information).

These three approaches provide interesting methods of collecting, processing and distributing information. However, they all tackle information management at the network layer, requiring significant changes to the network infrastructure. This is not feasible with current trends in technology.

However, if it is possible to achieve in-network processing at the application layer, then network infrastructure would not inhibit adoption of ICN. Building on the concepts such as:

1) information requests are expressed as an "interest"

2) information and its source are decoupled

3) information modeled as a recursive function of information

Service Oriented Architecture (SOA) is an architectural approach that decouples systems based on service interfaces [11]. There are clear lines of abstraction between the service provider and consumer. The systems are bound at run-time, meaning that many systems can be composed and re-composed dynamically at run-time. These principles of SOA can be used to create networks of applications which find data, process it into information and present it to the seeker.

\section{Cypher query language}

Open Cypher [4] is an SQL inspired graph query language first developed by Neo4J [12]. It is intended as an intuitive way of exploring graphs and executing graph algorithms such as shortest path. It supports full property graphs which allow properties to be associated with edges. It's notation uses a form of ASCII art to describe nodes and edges. A simple graph matching can be done as shown in Figure 1.

MATCH (nodea:nodeTA) - [edgeb:edgeTB] -> (nodec:nodeTC) RETURN nodea

Figure 1. A simple graph query matching two nodes related by an edge. Returned is a reference to the nodea entity. The nodea entity must match the relationship edgeb to nodec to satisfy the query.

Cypher also supports "WHERE" clause, and also property conditions within the "MATCH" clause. Additionally multiple "MATCH" clauses can be used within a single query in order to build the query.

\section{Arrowhead Framework}

The Arrowhead Framework (AF) [13] is a supporting technology for the solution. The AF is a SOA-based System of Systems (SoS) integration framework. The AF defines a service as provided by a system. Systems are then composed in SoS. A single system can provide and consume multiple services. Interactions between systems can only occur through these services. The AF provides the composition aspect of the solution. The expression builds a graph of systems and services. Each system is represented by a node, and each service as an edge between two systems. Systems can only interact if they have matching service provider and consumer interfaces. Matching service provider and consumer interfaces are described in the AF documentation structure [14].

\section{Proposed SOLUTION - EXPRESSiON OF INTEREST}

The proposed solution uses a declarative SQL like graph query to express interest. The query language is based on the Open Cypher graph query language. Cypher is selected as it has a natural manner of expressing service compositions based on System of System (SoS) approaches. Before describing the detail of the solution two alternatives are described here. In [15] a custom algebra was defined that can describe a graph composition based on service input/output matching. The benefit of this is that the graph can be directly applied to a graph of systems present in the ecosystem. Also, security and Quality of Service (QoS) aspects can be represented directly in the graph. However, expressing configuration of services (transformation of data) is not immediately clear.

In [8] an SQL styled declarative expression is used to describe the desired data. The SQL expression has been enhanced to allow data processing on returned data. However from the expression the mapping to services is unclear. The expressions are usually spread to all nodes and a middleware is used to process the query.

Here Cypher allows the both declarative queries, while maintaining a SOA, composition based, expression of interest for information. The Cypher query language is introduced in order to fully define its usage in the proposed solution.

\section{A. Expression}

An information query must define the source of the data, the processes, and the return format. In AF a service represents a function, including the parameter format, the processing and the return format. The system providing the service will either get raw data internally or process data from another system. Therefore, using Cypher to describe the system and service graph, all three aspects are defined. A simple example of a graph expression can be seen in Figure 2 Here the source of the information is a temperature sensor system (the far right system) and the processing is a threshold check. An intermediate system provides the thresholding service upon data retrieved the temperature service.

In this case, the application system need not perform thresholding internally and thus has reduced complexity. This may seem a trivial simplification, however, it means that its internal logic is not dependent on the data processing. It is possible to change the processing of data to a band-pass threshold without modification to the application system. A change like that 
MATCH () - [ts:ThresholdService] $->()$

$-[$ :TemperatureService $]->(\{1$ :chamber 1$\})$

WHERE ts.value > 60

RETURN ts.address

Figure 2. A simple Cypher query to express interest in the event of a chamber exceeding a threshold temperature.

could be due to a quality enhancement being identified by keeping temperature bounds within band.

\section{Proposed solution - Dynamic Service PROVISIONING}

So far the proposed solution has presented a way to describe interest in information. To process this information from raw data, there are certain functions required. For example, rather than raw temperature data, only values exceeding a threshold are required, or in the case of an IoT enabled wheel loader rather than data from individual wheel nodes, aggregated data is required. Once the information interest has been expressed it is parsed into a suitable composition of System of Systems (SoS). Parts of this composition are data processing systems such as:

1) Thresholding - reference triggers

2) Averaging - over time, over space

3) Filtering - low pass, high pass or band pass

4) Aggregating - list, array

5) Math - Summation, Subtraction, Multiplication, Division

6) Logic - OR, AND, XOR, NOT

7) Converting - converting measured units, i.e. $\mathrm{km}$ to miles. 8) etc

In this section a form of Dynamic Service Provisioning (DSP) is proposed. DSP allows for creation of SoS based on services which a created only when needed. This allows the ecosystem of services to dynamically grow and shrink as required by the context-aware systems. For example in Figure 3 the threshold system compares the temperature of a chamber to a reference level and pushes a notification when an event is triggered.

The systems in Figure 3 have several interfaces. Service "b" between the Orchestration system and the Threshold system contains 1) customization parameters and 2) target system addressing information. This is the systems composition interface. When a request has been received, if the threshold system is able to comply with the request, it will provision a sub-system which contains the interfaces for service "c" and "d". This is called dynamic system, which creates new services based on provisioning requests. The services are created for use in a particular system composition. Each of the subsystems is capable of handling different customization parameters. Furthermore, the individual subsystems are uniquely identified and have independent security controls and authentication. This provides many management advantages such as Quality of Service and logging. Figure 4 shows this as an architectural block diagram of a dynamic system structure.

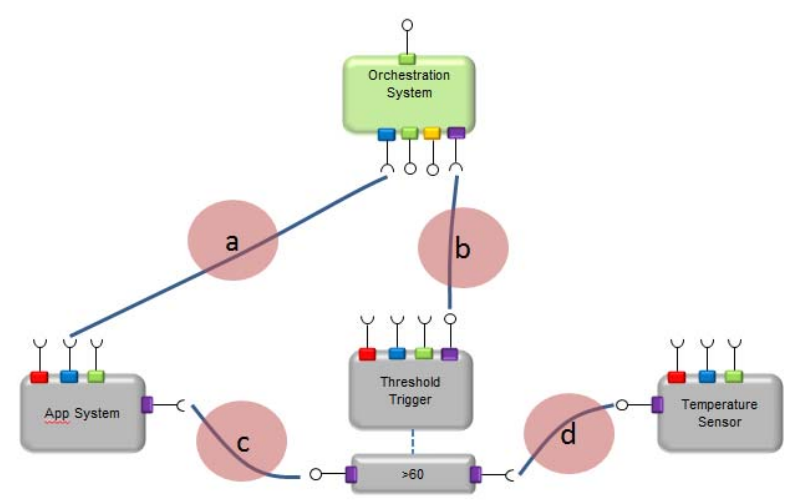

Figure 3. A basic service composition which creates context about the chamber temperature. If the chamber temperature crosses 60 degrees an event is pushed to a consuming system. The service labeled "a" contains the expression of interest as defined in Section III. The service labeled " $b$ " is the provisioning interface. Services "c" and "d" are the data exchange links which are part of normal operation.

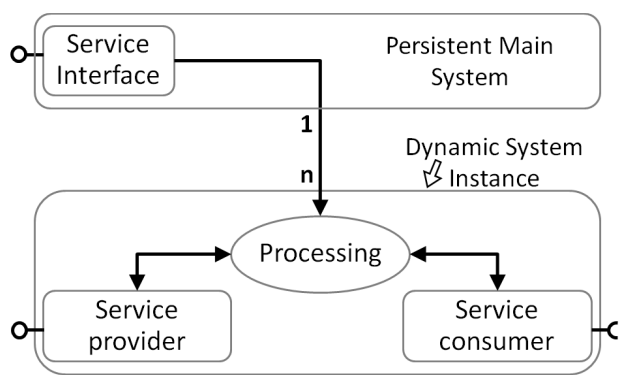

Figure 4. The block diagram of a dynamic system, able to provision new threshold interfaces based on request. Each sub-system is independent in terms of operation, but shares a single execution instance.

The dynamic provisioning interface will vary from system to system depending on the customization requirement. The variation of customization requirements is large and growing as new processes are added. SenML is a markup language used for reading and configuring sensor devices. It is lightweight and its loose definitions do not limit semantic meaning or limit its extension to new sensor information. Here we propose to use SenML as a means to send the customization information to the system. By using SenML it is possible to have a simple, reusable, library for serializing different parameters. As shown in Figure 5 the customization parameters for the threshold system are captured fully. Adapting this for another system is not difficult as the JSON parameter object " $n$ " is updated with the new parameter.

In response to such a request the processing system will give access details of the the newly provisioned service provider. Figure 6 shows an example of a response payload.

The dynamic service instance makes use of the Arrowhead framework authorization pattern. This means that access control by the data source allows ownership of the data to be maintained. Where mutual commercial agreement between two systems is not available, the dynamic system could act as a third party, so long there exists a commercial agreement 


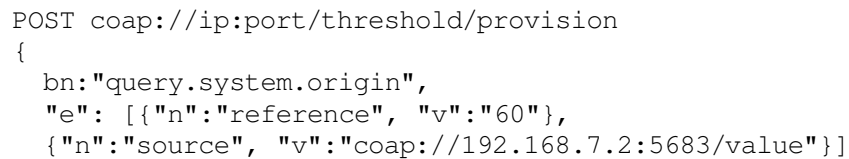

Figure 5. A JSON example request to provision a new interface for the threshold system. Here, SenML/JSON content type is used. "bn" is a basename and is the system who originated the query. "e" refers to the element array and each item of the element array are configuration parameters related for the specific processing system type. In this case CoAP is the communication protocol, but this could also be HTTP or MQTT

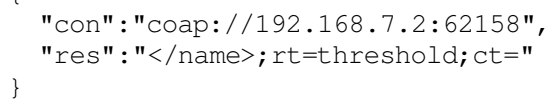

Figure 6. A JSON example response to a provision request.

between both systems and the dynamic system. This also holds for building a trust bridge where direct trust cannot be established, a Friend-of-a-friend. Furthermore, in some cases, the data holds more information than required by the context-aware system. In such cases, the context aware system need only have access rights to sub information and not the complete data. Hence, a dynamic system which has access rights to the full data, can process it and reduce the information content to only that which the context-aware system has rights to. This enables privacy preserving features of the information extraction architecture.

These advanced features are enabled because of the independence of the dynamic systems and their provisioned subsystems. It is also possible for multiple instances of a dynamic systems run by different operators to exist within a single network. Therefore, the paths available from the composition graph can be varied depending on the information and security requirement.

\section{WHEEL LOADER BALL BEARING MONITORING}

A pilot scenario from the Arrowhead project involves monitoring the vibrations, temperature and rotations of wheel loader ball bearings. Wheel loaders must be maintained in order to extend lifetime. Measuring wear, looking for anomalies and scheduling preventative maintenance are key objectives.

On a wheel loader with 4 wheels, there are 4 independent systems monitoring each wheel. Each system provides a service which when queried will return the ball bearing data presented in Figure 7.

The monitoring application could be designed to "seek out" each of the 4 sensor systems and begin query them for their data (publish/subscribe is also possible). This would need to be repeated for each wheel loader in service. This complexity is a by-product of the original mission of the monitoring application: 1) to schedule preventative maintenance and, 2) to notify if early end-of-life has occurred.

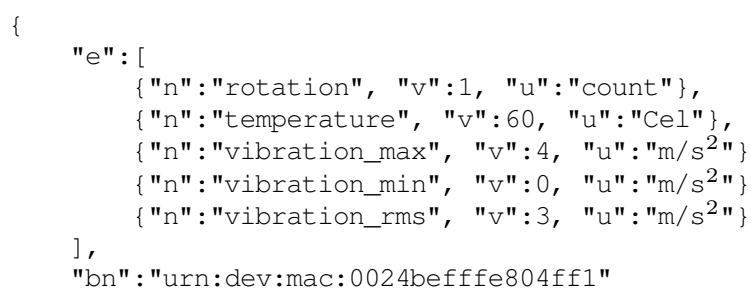

Figure 7. The wheel loader ball bearing sensor data returned when queried.

Using the proposed methods, it is possible to reduce application complexity. By allowing the SOA-based System of Systems to do the processing the application only need handle relevant information. In this case, the expression of interest for information is shown in Figure 8.

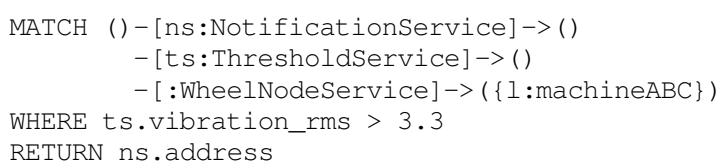

Figure 8. The expression of interesting using open Cypher query language and the proposed SOA-based description. Where "ns" is the notificationService, "ts" is the threshold service, "l" is the machine which the wheel loader node is on.

In this case first the ball bearing data must be collected and threshold tested, then the data must be aggregated for all wheels on a malfunction wheel loader, before being sent back to the monitoring application. This requires a dynamic threshold system and aggregation system to be provisioned in order to present the ball bearing sensor data. This composition is shown in Figure 9.

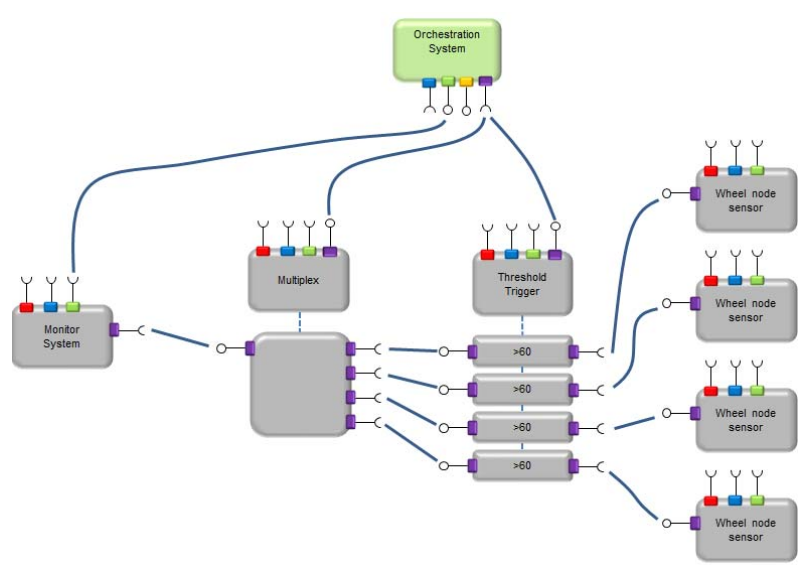

Figure 9. A diagram of the system composition which creates the information for the context-aware monitoring application. The blocks connected with dashed line are a single module, with the blocks above being persistent, while the blocks below being temporary.

This means that the information has been completely processed by standardized and reusable systems. It means that 
the monitoring application simply awaits notification by the composition and then schedules the maintenance.

\section{A. Implementation}

The objective of this implementation is to test that interface definitions and the proposal is sound. The base programming language is Java for applications systems. Using Java allowed for development on a windows machine and subsequent deployment to Linux distribution. Where Java is not suitable, i.e. embedded devices, implementation uses the $\mathrm{C}$ programming language.

This scenario has been implemented using a Beagle Bone Black (BBB) [16] to run the Arrowhead core systems, and the ball bearing sensor systems on the Mulle [17] boards. Using ContikiOS [18] and CoAP [19] server on each node, the services register with the Arrowhead service registry in the BBB. The monitoring application creates an interest for information request to the Arrowhead Orchestration engine, which performs a lookup on current ball bearing sensors and composes the threshold and aggregation systems. The monitoring application in this case was a stub application which simply recorded that the wheel loader required servicing or not.

\section{CONCLUSION}

Industry is looking to gain flexibility in growing and evolving their manufacturing systems. Applying Service Oriented Architecture (SOA) to System of Systems (SoS) engineering allows flexible networked embedded systems to participate in multilayer enterprise systems. In particular interest of reusable systems is that of information processing. There has been a trend to move away from centralized systems. This is due to the weaknesses of; 1) single-point-of-failure, 2) bottlenecks, 3 ) resistance to change and 4) costly maintenance.

This work has presented a novel approach for information extraction for context-aware systems in Industry 4.0. Contextaware computing when applied to SoS results in intelligent systems which can make decisions based changes to location, recipe, quality, availability, safety, and environment. This reduces, and in some cases removes, the requirement on centralized infrastructure for data processing and storage. It also helps to maintain soft real-time operation in the overall production line by enabling edge analytics. Centralized systems are used to manage overall objectives and to compose SoS. However, once the SoS is composed and the systems have the required knowledge, they can operate without further guidance from centralized systems.

The proposed solution was showcased on a demonstrator use case of a wheel loader ball bearing monitoring application.

This work will be continued to further refine and formalize the composition methodology. Building on the expression of interest for information and the dynamic systems proposed here, the composition engine must analyze the available composition graph to find the optimal shortest path.

In addition to the information requirement, the security overlay must be considered for the composition. This includes strict access-control and trust rules and more malleable trust which can be established dynamically.

\section{ACKNOWLEDGEMENT}

The authors would like to express our gratitude towards our partners within the Far-Edge project and the on going work within the Arrowhead Framework open project.

\section{REFERENCES}

[1] H. Lasi, P. Fettke, H.-G. Kemper, T. Feld, and M. Hoffmann, "Industry 4.0," Business \& Information Systems Engineering, vol. 6, no. 4, pp. 239-242, Aug 2014. [Online]. Available: https://doi.org/10.1007/s12599-014-0334-4

[2] B. Scholten, The road to integration : a guide to applying the ISA-95 standard in manufacturing. Research Triangle Park NC : ISA, 2007.

[3] "The Reference Architecture Model RAMI 4.0 and the Industrie 4.0 component - zvei.org," August 2017. [Online]. Available: https://www.zvei.org/en/subjects/industry-4-0/the-referencearchitectural-model-rami-40-and-the-industrie-40-component/

[4] "OpenCypher," May 2017. [Online]. Available: http://www.opencypher.org/

[5] "Arrowhead Framework Wiki," May 2016. [Online]. Available: https://forge.soa4d.org/plugins/mediawiki/wiki/arrowheadf/index.php/Main_Page

[6] G. D. Abowd, A. K. Dey, P. J. Brown, N. Davies, M. Smith, and P. Steggles, "Towards a better understanding of context and contextawareness," in International Symposium on Handheld and Ubiquitous Computing. Springer, 1999, pp. 304-307.

[7] A. S. Tanenbaum and M. v. Steen, Distributed Systems: Principles and Paradigms (2Nd Edition). Upper Saddle River, NJ, USA: Prentice-Hall, Inc., 2006.

[8] S. R. Madden, M. J. Franklin, J. M. Hellerstein, and W. Hong, "Tinydb: An acquisitional query processing system for sensor networks," ACM Trans. Database Syst., vol. 30, no. 1, pp. 122-173, Mar. 2005. [Online]. Available: http://doi.acm.org/10.1145/1061318.1061322

[9] M. A. Yaqub, S. H. Ahmed, S. H. Bouk, and D. Kim, InformationCentric Networks (ICN). Singapore: Springer Singapore, 2016, pp. 19-33.

[10] T. Braun, V. Hilt, M. Hofmann, I. Rimac, M. Steiner, and M. Varvello, "Service-centric networking," in 2011 IEEE International Conference on Communications Workshops (ICC), June 2011, pp. 1-6.

[11] T. Erl, SOA Design Patterns, 1st ed. Upper Saddle River, NJ, USA: Prentice Hall PTR, 2009.

[12] "Neo4j Graph Database," May 2017. [Online]. Available: https://neo4j.com/

[13] J. Delsing, IoT Automation: Arrowhead Framework. CRC Press, 2017.

[14] F. Blomstedt, L. L. Ferreira, M. Klisics, C. Chrysoulas, I. M. de Soria, B. Morin, A. Zabasta, J. Eliasson, M. Johansson, and P. Varga, "The arrowhead approach for soa application development and documentation," in Industrial Electronics Society, IECON 2014 - 40th Annual Conference of the IEEE, Oct 2014, pp. 2631-2637.

[15] S. V. Hashemian and F. Mavaddat, "A graph-based framework for composition of stateless web services," in 2006 European Conference on Web Services (ECOWS'06), Dec 2006, pp. 75-86.

[16] "Beagle Bone Black," May 2017. [Online]. Available: https://beagleboard.org/black

[17] "Eistec AB," May 2017. [Online]. Available: http://www.eistec.se/

[18] A. Dunkels, B. Gronvall, and T. Voigt, "Contiki - a lightweight and flexible operating system for tiny networked sensors," in Local Computer Networks, 2004. 29th Annual IEEE International Conference on, Nov 2004, pp. 455-462.

[19] C. Bormann, A. Castellani, and Z. Shelby, "Coap: An application protocol for billions of tiny internet nodes," Internet Computing, IEEE, vol. 16, no. 2, pp. 62-67, March 2012. 